\title{
Spectroscopic analysis of lithium terbium tetrafluoride
}

\section{Christensen, H.P.}

\section{Published in:}

Physical Review B

Link to article, DOI:

10.1103/PhysRevB.17.4060

Publication date:

1978

\section{Document Version}

Publisher's PDF, also known as Version of record

Link back to DTU Orbit

Citation (APA):

Christensen, H. P. (1978). Spectroscopic analysis of lithium terbium tetrafluoride. Physical Review B, 17(10), 4060-4068. https://doi.org/10.1103/PhysRevB.17.4060

\section{General rights}

Copyright and moral rights for the publications made accessible in the public portal are retained by the authors and/or other copyright owners and it is a condition of accessing publications that users recognise and abide by the legal requirements associated with these rights.

- Users may download and print one copy of any publication from the public portal for the purpose of private study or research.

- You may not further distribute the material or use it for any profit-making activity or commercial gain

- You may freely distribute the URL identifying the publication in the public portal

If you believe that this document breaches copyright please contact us providing details, and we will remove access to the work immediately and investigate your claim 


\title{
Spectroscopic analysis of lithium terbium tetrafluoride
}

\author{
H. P. Christensen \\ Department of Electrophysics, The Technical University of Denmark, DK-2800 Lyngby, Denmark \\ (Received 12 December 1977)
}

\begin{abstract}
The absorption spectra of $\mathrm{Tb}^{3+}$ in $\mathrm{LiTbF}_{4}$ have been recorded in the spectral interval from 4000 to 25000 $\mathrm{cm}^{-1}$ and for temperatures between 2.3 and $150 \mathrm{~K}$. This covers the transitions from the ground multiplet ${ }^{7} F_{6}$ to the multiplets ${ }^{7} F_{3},{ }^{7} F_{2},{ }^{7} F_{1},{ }^{7} F_{0}$, and ${ }^{5} D_{4}$. The transitions were predominantly of electric-dipole nature, but small contributions of magnetic-dipole nature were seen. The crystal-field splitting was temperature dependent-the reason for this is not completely understood. No experimental evidence for a crystallographic phase transition was found. The energy levels of the ground ${ }^{7} \mathrm{~F}$ term were calculated by diagonalizing an effective spin-orbit and crystal-field Hamiltonian in an $L S$ basis. $H=\sum \lambda_{i}(\overrightarrow{\mathrm{L}} \cdot \overrightarrow{\mathrm{S}})^{i}+\sum \alpha_{i} \sum B_{i m} O_{i m}$, where the parameters $\lambda_{i}$ are functions of the spin-orbit parameter $\zeta$ and the Slater parameter $F_{2}$. The $O_{i m}$ and $\alpha_{i}$ are Racah operators and reduced matrix elements, respectively. The rare-earth site in $\mathrm{LiTbF}_{4}$ possesses $S_{4}$ symmetry, which allows six crystal-field parameters. $\zeta$ and the six $B_{i m}$ were varied to obtain the best agreement with the experimentally observed levels. Keeping $F_{2}=434 \mathrm{~cm}^{-1}$ fixed, a fit with a standard deviation of $12 \mathrm{~cm}^{-1}$ was obtained at $10 \mathrm{~K}$ with the following parameters: $\zeta=1698 \mathrm{~cm}^{-1}, B_{20}=445 \mathrm{~cm}^{-1}$, $B_{40}=-761 \mathrm{~cm}^{-1}, B_{44}=1120 \mathrm{~cm}^{-1}, B_{60}=4 \mathrm{~cm}^{-1}$, and $B_{64}=761+i 609 \mathrm{~cm}^{-1}$. Although the ground $L S$ term of $\mathrm{Tb}^{3+}$ is rather isolated, the term mixing is significant, which is also the case for the multiplet mixing. Even for the ground multiplet the $J$ mixing cannot be ignored.
\end{abstract}

\section{INTRODUCTION}

The characteristics of the tripositive rare-earth ions $\left(R^{3+}\right)$ doped in $\mathrm{LiYF}_{4}$ have been studied in numerous works. The main reason for this is the interesting optical properties of these systems.

For the rare earth heavier than $\mathrm{Sm}$ it is possible to grow LiY ${ }_{1-x} R_{x} \mathrm{~F}_{4}$ crystals for any value of $x$. Until now, however, most of the attention has been focused on the crystals dilute in $R$ content. Only recently, crystals dense in $R$ have been investigated, and it has mainly been the magnetic properties which have been examined. ${ }^{1-6}$ Especially. the features of the systems in the temperature region where the ordered phase appears ${ }^{7-10}$ have been investigated.

Approximate crystal-field parameters have been determined from susceptibility and magnetization measurements, ${ }^{2,5}$ but only a few direct spectroscopic observations of the crystal-field splitting of the energy levels of $R^{3+}$ in $\mathrm{Li} R \mathrm{~F}_{4}$ have been made..$^{8,11}$

The reason why works on crystals dilutely doped with $R$ have been predominant is that for optical applications one is often limited to such systems due to destructive $R-R$ interaction, i.e., concentration quenching of the fluorescence. There are, however, exceptions, one of which is the $\mathrm{LiY}_{1-x} R_{x} \mathrm{~F}_{4}$ system. Long-lived ( $5 \mathrm{msec}$ ) green fluorescence has been reported ${ }^{12}$ from $\mathrm{Tb}^{3+}$ in $\mathrm{LiY}_{0_{\circ} 5} \mathrm{~Tb}_{0.25} \mathrm{Gd}_{0_{\circ} 25} \mathrm{~F}_{4}$ and measurements ${ }^{13}$ on $\mathrm{Tb}^{3+}$ in $\mathrm{LiTbF}_{4}$ show that the lifetime of the green light is still longer than $1 \mathrm{msec}$.

Therefore, it is of interest to know in detail the energy-level scheme of $R^{3+}$ in the dense crystal, and here I report on the results for the whole ${ }^{7} F$ term and the lowest ${ }^{5} \mathrm{D}_{4}$ multiplet for the case of $\mathrm{Tb}^{3 *}$.

\section{THEORY}

A. Free $\mathrm{Tb}^{3+}$ ion

The ground configuration of the free $\mathrm{Tb}^{3+}$ ion has eight $4 f$ electrons outside closed orbitals. The next configuration, which is a $4 f^{7} 5 d$, is approximately $60000 \mathrm{~cm}^{-1}$ above the $4 f^{8}$ configuration according to Dieke. ${ }^{14}$ Thus, for the lowest levels of the ground configuration, the configuration mixing is small, and for these levels it is a good approximation to do all the energy calculations within the $4 f^{8}$ configuration.

In this configuration the ground $L S$ term is the rather isolated ${ }^{7} F$. The width of the ${ }^{7} F$ term is $6000 \mathrm{~cm}^{-1}$ and the next term, which is a ${ }^{5} D$, is situated about $20000 \mathrm{~cm}^{-1}$ above. In ${ }^{5} \mathrm{D}$ the lowest multiplet ${ }^{5} D_{4}$ is rather isolated and acts as the upper fluorescence level in $\mathrm{Tb}^{3+}$. While for the ${ }^{7} F$ term the term mixing is small, this is not true for the other terms. The configuration extends to about $150000 \mathrm{~cm}^{-1}$, and above $25000 \mathrm{~cm}^{-1}$ the classification of the levels is somewhat uncertain.

Although the term mixing for ${ }^{7} F$ is moderate, the Lande interval rule for the spin-orbit splitting is not well fulfilled, so it is necessary to introduce even this slight mixing in the calculations. The $L$ and $S$ quantum numbers are in this way no longer really good quantum numbers contrary to $J$. The spin-orbit coupling splits the ground term in seven 
TABLE I. Crystal-field splittings of $J$ multiplets in $S_{4}$ symmetry.

\begin{tabular}{ccccc}
\hline$J$ & $\Gamma_{1}$ & $\Gamma_{2}$ & $\Gamma_{3}$ & $\Gamma_{4}$ \\
\hline 0 & 1 & 0 & 0 & 0 \\
1 & 1 & 0 & 1 & 1 \\
2 & 1 & 2 & 1 & 1 \\
3 & 1 & 2 & 2 & 2 \\
4 & 3 & 2 & 2 & 2 \\
5 & 3 & 2 & 3 & 3 \\
6 & 3 & 4 & 3 & 3 \\
\hline
\end{tabular}

multiplets with $J=0,1, \ldots, 6$, where ${ }^{7} F_{6}$ is the ground multiplet.

\section{B. $\mathrm{Tb}^{3+}$ ion in a crystal with $S_{4}$ symmetry}

$\mathrm{LiTbF}_{4}$ crystallizes in the tetragonal scheelite structure. The $\mathrm{Tb}^{3+}$ ion is on a site having $S_{4}$ symmetry with respect to the crystal as a whole, but almost $D_{2 d}$ symmetry with respect to the eight nearest-neighbor $\mathrm{F}^{*}$ ions. In $S_{4}$ symmetry the $J$ multiplets split up as shown in Table I, where $\Gamma_{1}$, $\Gamma_{2}, \Gamma_{3}$, and $\Gamma_{4}$ refer to the notation of Koster et al. ${ }^{15}$

The $\Gamma_{3}$ and $\Gamma_{4}$ representations are related by time-reversal symmetry, and the eigenvalues for states transforming according to these representations will therefore be degenerate. In the following they will be named by $\Gamma_{3,4}$.

The crystal field mixes the multiplets slightly (less than $10 \%$ ), causing $J$ to be not exactly a good quantum number. Therefore, it is necessary to take $J$ mixing into account in the calculations of the energies of the levels. This, however, does not change the transformation properties of the associated states.

\section{Selection rules for $S_{4}$ symmetry}

The two most significant contributions to the transitions between the levels are the electric and magnetic dipole interaction with the applied electromagnetic radiation. The electric dipole transitions are parity forbidden within a single configuration, so they occur only due to configuration mixing. Still, for the rare earths they are, in . general, stronger than the magnetic dipole transitions.

Thus, calculation of transition strengths requires knowledge of configuration mixing, which is difficult to determine. On the contrary, separation of allowed and forbidden transitions can be done entirely on group-theoretical grounds. The selection rules for electric and magnetic dipole transitions in $S_{4}$ symmetry are given in Table II.
TABLE II. Selection rules in $S_{4}$ symmetry: (a) electric dipole transitions, (b) magnetic dipole transitions; The $\pi$ spectrum has the electric polarization parallel to the $c$ axis. The $\sigma$ spectrum has the electric polarization perpendicular to the $c$ axis.

\begin{tabular}{cccc}
\hline \hline (a) & $\Gamma_{1}$ & $\Gamma_{2}$ & $\Gamma_{3,4}$ \\
\hline$\Gamma_{1}$ & & $\pi$ & $\sigma$ \\
$\Gamma_{2}$ & $\pi$ & & $\sigma$ \\
$\Gamma_{3,4}$ & $\sigma$ & $\sigma$ & $\pi$ \\
(b) & $\Gamma_{1}$ & $\Gamma_{2}$ & $\Gamma_{3,4}$ \\
\hline$\Gamma_{1}$ & $\sigma$ & & $\pi$ \\
$\Gamma_{2}$ & & $\sigma$ & $\pi$ \\
$\Gamma_{3,4}$ & $\pi$ & $\pi$ & $\sigma$ \\
\hline \hline
\end{tabular}

D. Model Hamiltonian

As stated in Secs. I A-IC the configuration mixing is neglected in the calculations, whereas the mixing of $L S$ terms and of $J$ multiplets is taken into account by using a generalized spin-orbit Hamiltonian and by diagonalizing the entire $L S$ matrix of the ground term.

Following Karayianis ${ }^{16}$ the effective spin-orbit operator to order $p$ is given by

$$
H_{\mathrm{so}}=\sum_{i=1}^{p} \lambda_{i}(\overrightarrow{\mathrm{L}} \cdot \overrightarrow{\mathrm{S}})^{i}
$$

where the parameters $\lambda_{i}$ are given by

$$
\lambda_{i}=\zeta \sum_{m=i}^{D}\left(\frac{\zeta}{F_{2}}\right)^{m=1} \Lambda_{i m}(N),
$$

where $\zeta$ is the spin-orbit parameter and $F_{2}$ is a Slater parameter. $\Lambda_{i m}(N)$, where $N$ is the number of $f$ electrons, are reduced matrix elements. They depend on the radial part of the wave functions only through the ratios $F_{4} / F_{2}$ and $F_{6} / F_{2}$, which are wave function insensitive. The parameters $\Lambda$ are given by Karayianis. ${ }^{16}$

The crystal-field operator is given by

$$
H_{c}=\sum_{i} \alpha_{i}(L) \sum_{m} B_{i m} O_{i m}(L)
$$

where $O_{i m}(L)$ are Racah operator equivalents and $\alpha_{i}(L)$ are reduced matrix elements. In $S_{4}$ symmetry the only nonvanishing crystal-field parameters are $B_{20}, B_{40}, B_{44}, B_{60}$, and $B_{64}$, where $B_{44}$ and $B_{64}$ are complex. By proper choice of the coordinate system one can make one of them-for example, $B_{44}$-real, leaving six crystal-field parameters to be determined. 


\section{EXPERIMENTAL PROCEDURE}

A. Crystal preparation

Single crystals of pure $\mathrm{LiTbF}_{4}$ and of $\mathrm{LiYF}_{4}$ doped with $10 \% \mathrm{~Tb}$ were grown by spontaneous crystallization from the melt as described by Laursen and Holmes. ${ }^{17}$ The crystals were orientated by $\mathrm{x}$-ray technique. Slabs about $1 \mathrm{~mm}$ thick were cut with faces perpendicular to an $a$ axis for the polarized spectra and with faces perpendicular to the $c$ axis for the axial spectrum. The slabs were mounted so that the angle between the crystal axis and the polarization axis was less than $2^{\circ}$ (in fact, the spectra were rather insensitive to small deviations between the directions of the polarization and the crystal axes).

\section{B. Apparatus}

The absorption spectra were recorded using a modified Zeiss MM12 double monochromator. In the relevant spectral region it was possible to achieve a spectral resolution of $6 \mathrm{~cm}^{-1}$. A Glan prism was used as polarizer.

For low-temperature measurements two cryostats were available. The first one, where the crystal was placed directly in the pumped liquid helium, was used for temperatures below $4.2 \mathrm{~K}$. The other one, where the crystal was placed in a stream of helium gas, was used for temperatures above $4.2 \mathrm{~K}$. In the first cryostat the temperature was determined from the He pressure. In the second the temperature was measured with an $\mathrm{Au}-$ Fe vs Cromel thermocouple, which had a resolution of $1 \mathrm{~K}$. The temperature stability of the flow cryostat was $\pm 1 \mathrm{~K}$.

\section{Absorption spectra}

The absorption spectra for polarized light for $\mathrm{Tb}^{3+}$ in $\mathrm{LiTbF}_{4}$ were recorded in the region from 4000 to $25000 \mathrm{~cm}^{-1}(2.5-0.4 \mu \mathrm{m})$, which covers the transitions from the ${ }^{7} F_{6}$ ground multiplet to the four highest multiplets of the ground term $(J=$ $0,1,2,3)$ and to the lowest ${ }^{5} D_{4}$ multiplet.

The spectra were recorded for different temperatures between 2.3 and $150 \mathrm{~K}$. However, for temperatures higher than $100 \mathrm{~K}$, the positions of most of the lines were difficult to determine because the spectra were smeared out due to overlapping with many new lines.

\section{Transitions within the ground term}

At $10 \mathrm{~K}$, which is well above the Curie point $T_{C}$ $=2.87 \mathrm{~K},{ }^{7}$ only the ground state, which is a quasidoublet made up of two levels transforming according to $\Gamma_{2}$ of $S_{4}$ (Laursen and Holmes ${ }^{17}$ ), is populated. As shown in Fig. 1 one finds at this tempera-

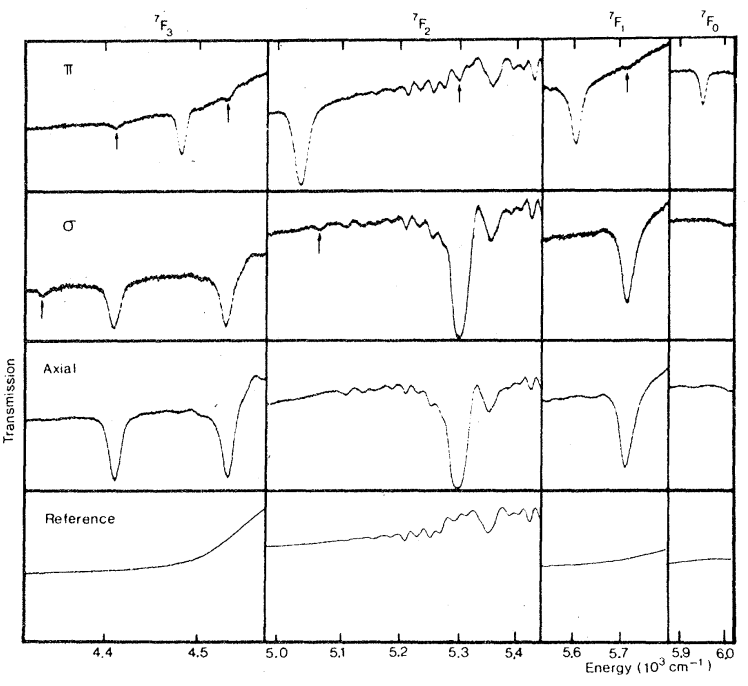

FIG. 1. Absorption spectra at $10 \mathrm{~K}$ for transition from the ground state to ${ }^{7} F_{3},{ }^{7} F_{2},{ }^{7} F_{1}$, and ${ }^{7} F_{0}$ of $\mathrm{Tb}^{3+}$ in $\mathrm{LiTbF}_{4}$. The $\pi$-polarized, the $\sigma$-polarized, the axial, and the reference spectrum are shown. The arrows indicate the magnetic dipole transitions.

ture for each of the two polarizations four strong absorption lines in the spectral region, where transitions to the ${ }^{7} F_{0}-{ }^{7} F_{3}$ multiplets are expected.

This is in agreement with the transitions being of electric dipole nature. $\pi$ transitions from the $\Gamma_{2}$ ground levels to $\Gamma_{1}\left({ }^{7} F_{0}\right), \Gamma_{1}\left({ }^{7} F_{1}\right), \Gamma_{1}\left({ }^{7} F_{2}\right)$, and $\Gamma_{,}\left({ }_{1}^{7} F_{3}\right)$ lead to four lines. $\sigma$ transitions from $\Gamma_{2}$ to $\Gamma_{3,4}\left({ }^{7} F_{1}\right), \Gamma_{3,4}\left({ }^{7} F_{2}\right)$, and two $\Gamma_{3,4}\left({ }^{7} F_{3}\right)$ give four lines, while the $\Gamma_{2}$ to $\Gamma_{2}$ transitions are not electric dipole allowed in $S_{4}$ symmetry.

In addition to these strong lines there are also some weak lines in the spectra. The transitions from $\Gamma_{2}$ to $\Gamma_{3,4}$ can be seen in the $\pi$ spectrum, whereas the $\Gamma_{2}$ to $\Gamma_{1}$ transitions cannot be seen in the $\sigma$ spectrum. This indicates that there are also magnetic dipole transitions present-and that the lines are not due to misorientation of the crystal (compare with Table II). The magnetic dipole transitions from $\Gamma_{2}$ to $\Gamma_{2}$ are allowed in the $\sigma$ spectrum. Experimentally one also finds two weak lines in the $\sigma$ spectrum corresponding to two of the four possible $\Gamma_{2}$ to $\Gamma_{2}$ transitions. The two other lines should be found in regions, where they are difficult to recover due to the strong lines and noise. A few other very weak lines in the spectra must be due to impurities in the crystal.

As a verification of the nature of the transitions, the axial spectrum was recorded. In the axial spectrum one should find the electric dipole transitions of the $\sigma$ spectrum and the magnetic dipole transitions of the $\pi$ spectrum. Thus, in the axial spectrum only $\Gamma_{2}$ to $\Gamma_{3,4}$ transitions are to be seen. This is what is found experimentally (see 
TABLE III. Experimental positions of the ${ }^{7} F$ levels, which it has been possible to determine, and calculated positions of all the levels. All values are in $\mathrm{cm}^{-1}$.

\begin{tabular}{|c|c|c|c|c|c|c|c|}
\hline \multirow[b]{2}{*}{$J$} & \multirow[b]{2}{*}{$\Gamma$} & \multirow[b]{2}{*}{$10 \mathrm{~K}$} & \multicolumn{3}{|c|}{ Experimental values at $T=$} & \multirow[b]{2}{*}{$100 \mathrm{~K}$} & \multirow{2}{*}{$\begin{array}{c}\text { Calculated values } \\
\qquad(10 \mathrm{~K})\end{array}$} \\
\hline & & & $40 \mathrm{~K}$ & $50 \mathrm{~K}$ & $60 \mathrm{~K}$ & & \\
\hline 0 & 1 & 5917 & 5917 & 5915 & 5913 & 5909 & 5923 \\
\hline \multirow[t]{2}{*}{1} & 3,4 & 5690 & 5689 & 5688 & 5685 & 5681 & 5714 \\
\hline & 1 & 5592 & 5591 & 5590 & 5588 & 5586 & 5603 \\
\hline \multirow[t]{4}{*}{2} & 2 & 5385 & & & & & 5371 \\
\hline & 3,4 & 5285 & 5284 & 5283 & 5282 & 5278 & 5285 \\
\hline & 2 & 5067 & & & & & 5064 \\
\hline & 1 & 5035 & 5035 & 5034 & 5034 & 5033 & 5024 \\
\hline \multirow[t]{5}{*}{3} & 2 & 4532 & & & & & 4536 \\
\hline & 3,4 & 4524 & 4524 & 4522 & 4522 & 4516 & 4528 \\
\hline & 1 & 4473 & 4473 & 4472 & 4472 & 4470 & 4478 \\
\hline & 3,4 & 4406 & 4406 & 4405 & 4404 & 4404 & 4404 \\
\hline & 2 & 4333 & & & & & 4324 \\
\hline \multirow[t]{7}{*}{4} & 1 & & & & & & 3845 \\
\hline & 3,4 & & & & & & 3630 \\
\hline & 2 & & & & & & 3572 \\
\hline & 1 & & & & & & 3539 \\
\hline & 3,4 & & & & & & 3400 \\
\hline & 2 & & & & & & 3399 \\
\hline & 1 & & & & & & 3325 \\
\hline \multirow[t]{8}{*}{5} & 2 & & & & & & 2403 \\
\hline & 3,4 & & & & & & 2390 \\
\hline & 1 & & & & & & 2339 \\
\hline & 3,4 & & & & & & 2162 \\
\hline & 1 & & & & & & 2148 \\
\hline & 1 & & & & & & 2130 \\
\hline & 3,4 & & & & & & 2089 \\
\hline & 2 & & & & & & 2087 \\
\hline \multirow[t]{10}{*}{6} & 2 & & & & & & 401 \\
\hline & 3,4 & & & & & & 390 \\
\hline & 1 & & & & & & 376 \\
\hline & 1 & & & 217 & & & 223 \\
\hline & 3,4 & & & 166 & & & 175 \\
\hline & 2 & & & 136 & & & 135 \\
\hline & 1 & & & 124 & & & 119 \\
\hline & $3,4-$ & & & 107 & & & 108 \\
\hline & 2 & & & 0 & & & 2 \\
\hline & 2 & 0 & 0 & 0 & 0 & 0 & 0 \\
\hline
\end{tabular}

Fig. 1).

At higher temperatures more levels of the ground multiplet become populated. Already at about $25 \mathrm{~K}$ the transitions from the first excited level $\left(\Gamma_{3,4}\right)$ can be seen in the spectra, even if the relative population of this level is only around 3 $\times 10^{-3}$. As the temperature is further increased, more and more lines appear in the spectra. By analyzing the spectra at temperatures up to $100 \mathrm{~K}$, it has been possible to determine the energies of the seven lowest levels of the ground multiplet and of the two remaining $\Gamma_{2}$ levels of ${ }^{7} F_{3}$ and ${ }^{7} F_{2}$. The positions of the experimentally determined lines of the ground $L S$ term are listed in Table III.

The experimental linewidths of the electric dipole transitions from the ground quasidoublet are $10-20 \mathrm{~cm}^{-1}$, with an instrumental resolution of about $6 \mathrm{~cm}^{-1}$. The lines are not much broader at higher temperatures, but the lines are getting weaker due to depopulation of the ground state, and some of them become disturbed by new lines.

Some of the lines, which according to the theory 


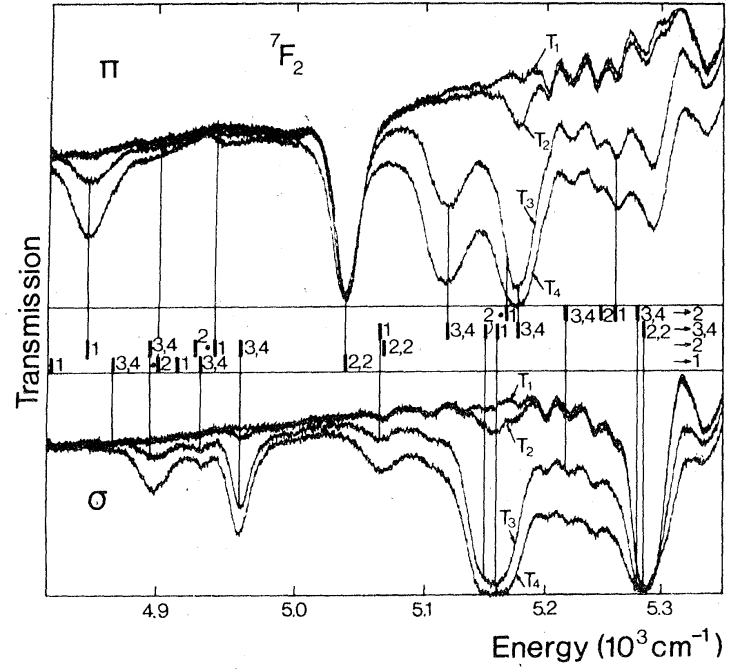

FIG. 2. $\pi$ - and $\sigma$-polarized absorption spectra for transition from ${ }^{7} F_{6}$ to ${ }^{7} F_{2}$ of $\mathrm{Tb}^{3+}$ in $\mathrm{LiTbF}_{4}$ at four different temperatures. $T_{1}=10 \mathrm{~K}, T_{2}=30 \mathrm{~K}, T_{3}=50 \mathrm{~K}$, and $T_{4}=70 \mathrm{~K}$. The seven lowest levels of ${ }^{7} F_{6}$ at $50 \mathrm{~K}$ are inserted in the figure with the ground $2 \times \Gamma_{2}$ coinciding with the low-temperature lines to explain the origin of the warm-up lines. $3,4 \rightarrow 2$ means the position where a transition from a $\Gamma_{3,4}$ level of ${ }^{7} F_{6}$ to a $\Gamma_{2}$ level of ${ }^{7} F_{2}$ should be found, etc. The indicated transitions are those allowed by electric dipole interaction in $S_{4}$ symmetry. Those marked with a dot are forbidden in $D_{2 d}$ symmetry.

in principle should be present, are experimentally absent. This, however, may be due to low transition probability. It is more important that no strong inexplicable lines are experimentally found. As an example, the spectra of the transitions from the excited states to ${ }^{7} F_{2}$ are shown in Fig. 2.

As mentioned earlier, the rare-earth ion is on a site of nearly $D_{2 d}$ symmetry, in which some $S_{4}$ symmetry-allowed transitions are forbidden. The two levels of the ground quasidoublet transform in $D_{2 d}$ like $\Gamma_{3}$ and $\Gamma_{4}$, respectively (notation of Koster et $\left.a l .{ }^{15}\right)$. Because both levels are well populated, there will be no changes in the selection rules corresponding to transitions from the ground state.

At higher temperatures, however, some of the transitions would be forbidden in $D_{2 d}$ symmetry. These are marked with a dot in Fig. 2. It is seen that none of these lines is strong, but some of them are definitely present above $50 \mathrm{~K}$. Below 50 $\mathrm{K} D_{2 d}$ symmetry cannot be excluded on basis of the present data.

The spectra recorded as a function of temperature show that the crystal-field-split multiplets are contracting as the temperature is increased, and do so by an amount much larger than expect-

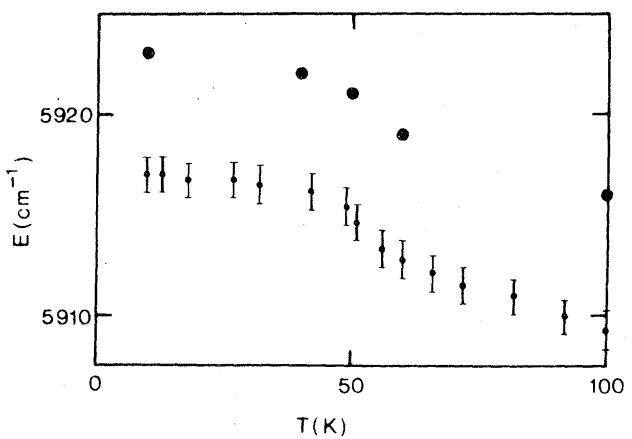

FIG. 3. Position of the transition from the ground state to ${ }^{7} F_{0}$ of $\mathrm{Tb}^{3+}$ in $\mathrm{LiTbF}_{4}$ as a function of the temperature. Data points with error bars are experimental values. are calculated values. The error bars indicate the relative uncertainty. The absolute uncertainty of the lines is $5 \mathrm{~cm}^{-1}$.

ed from the thermal expansion of the lattice. In addition to this smooth temperature contraction of the multiplets, it seems that the whole term contracts slightly $\left(2-3 \mathrm{~cm}^{-1}\right)$ in a small temperature region around $50 \mathrm{~K}$. As an example, the temperature dependence of the ${ }^{7} F_{0}$ line is shown in Fig. 3.

The temperature dependence of the multiplets is small below $50 \mathrm{~K}$, but from 50 to $100 \mathrm{~K}$ it is as large as $0.1 \mathrm{~cm}^{-1} / \mathrm{K}$ for some of the lines. This is equivalent to a contraction of the crystal-field splitting of about $0.03 \% / \mathrm{K}$. This strong temperature dependence is in agreement with the shift of the two lines of the ground multiplet seen by Holmes et $a l .^{2}$ in neutron scattering.

For those few lines, which it has been possible to follow from 100 to $150 \mathrm{~K}$, it seems that the temperature dependence is still present with about the same strength as below $100 \mathrm{~K}$. It has been checked that the observed temperature effect is not due to strain induced by the mounting of the crystal.

The contraction could be due to a second-order phase transitions, but experimentally there is no evidence of the presence of a phase with lower symmetry - which in this case should be $C_{2}$, where the $\Gamma_{3,4}$ degeneracy is removed and the $\Gamma_{1}$ to $\Gamma_{1}$ and $\Gamma_{2}$ to $\Gamma_{2}$ (in $S_{4}$ notation) electric-dipole transitions are allowed in the $\sigma$ spectrum.

As a supplement, the line shifts for $10 \% \mathrm{~Tb}$ in $\mathrm{LiYF}_{4}$ were examined. It was found that the multiplet contraction was much weaker $\left(<0.02 \mathrm{~cm}^{-1} / \mathrm{K}\right)$ than for the dense crystal, and the term contraction at $50 \mathrm{~K}$ was not detectable $\left(<1 \mathrm{~cm}^{-1}\right)$.

To study the effect of the magnetic ordering on the absorption spectra, the spectra were recorded down to $2.3 \mathrm{~K}$. Qualitatively, there was no change in the spectra, but for some of the lines there were line shifts in the temperature interval from 


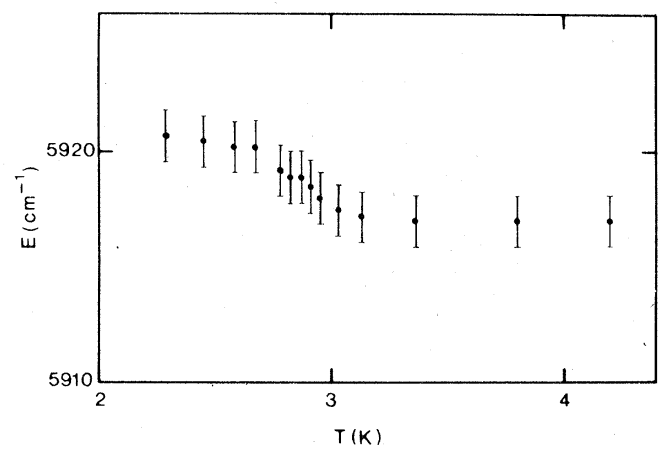

FIG. 4. Experimental position of the transition from the ground state to ${ }^{7} F_{0}$ of $\mathrm{Tb}^{3+}$ in $\mathrm{LiTbF}_{4}$ as a function of the temperature around $T_{C}$, where $\mathrm{LiTbF}_{4}$ order magnetically.

2.7 to $3.0 \mathrm{~K}$. This is in agreement with the fact that the crystal orders ferromagnetically below $2.87 \mathrm{~K}^{7}$ As shown in Fig. 4 the shift of the line corresponding to absorption to ${ }^{7} F_{0}$ is $3.5 \pm 1 \mathrm{~cm}^{-1}$ at $2.3 \mathrm{~K}$. The ordering causes the ground quasidoublet to split proportional to the magnetization into an $M_{J}=+6$ and an $M_{J}=-6$ level, of which only the lowest is well populated.

For an Ising system the shift at $0 \mathrm{~K}$ should be equal to $k_{B} \theta$, where $\theta$ is the asymptotic paramagnetic Curie temperature, assuming that in the ordered phase the domains are long thin needles along the $c$ axis. Holmes et al. ${ }^{1}$ give $\theta=3.60$ $\pm 0.1 \mathrm{~K}\left(\theta_{\mathrm{di} \mathrm{polar}}=3.97 \mathrm{~K}\right)$ and thus a shift of $2.5 \pm 0.1$ $\mathrm{cm}^{-1}$ is expected. The observed shift seems somewhat higher than this, similar to what has been

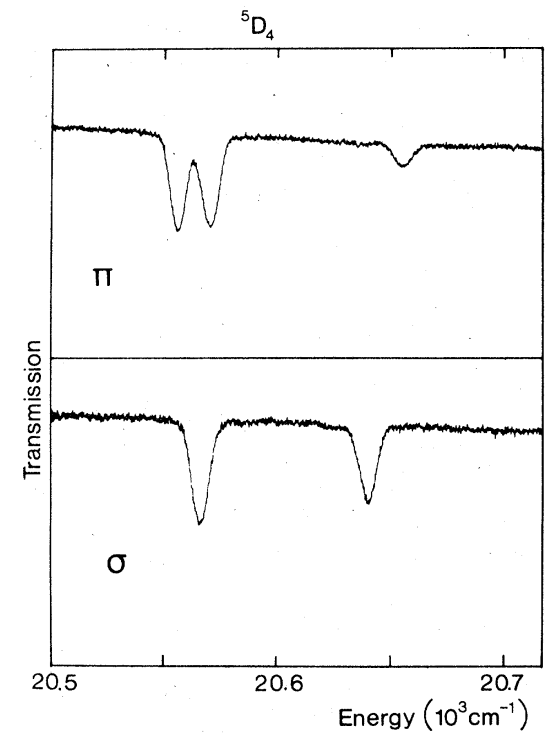

FIG. 5. $\pi$ - and $\sigma$-polarized absorption spectra at $10 \mathrm{~K}$ for the transitions from the ground state to ${ }^{5} D_{4}$ of $\mathrm{Tb}^{3+}$ in $\mathrm{LiTbF}_{4}$.
TABLE IV. Experimental positions in $\mathrm{cm}^{-1}$ of the ${ }^{5} D_{4}$ levels :

\begin{tabular}{ccc}
\hline \hline \multirow{J}{*}{$J$} & $\Gamma$ & $\begin{array}{c}\text { Experimental values } \\
(10 \mathrm{~K})\end{array}$ \\
\hline \multirow{4}{*}{4} & 2 & 20666 \\
& 1 & 20654 \\
& 3,4 & 20641 \\
2 & 20589 \\
& 1 & 20580 \\
& 3,4 & 20571 \\
& 1 & 20561 \\
\hline
\end{tabular}

found in $\mathrm{LiHoF}_{4}$ (Battison et al..$^{9}$ ) in which case the deviation might be explained by the large hyperfine interaction in $\mathrm{Ho}^{3+}$. This is not possible in the case of $\mathrm{Tb}^{3+}$ in $\mathrm{LiTbF}_{4}$, where the total hf splitting is $0.3 \mathrm{~cm}^{-1}$.

\section{Transitions to the ${ }^{5} \mathrm{D}_{4}$ multiplet}

In the region around $20600 \mathrm{~cm}^{-1}$ three lines in the $\pi$ spectrum and two lines in the $\sigma$ spectrum were found at low temperatures, as shown in Fig. 5. They are due to transitions from the ground state to the three $\Gamma_{1}$ levels and two $\Gamma_{3,4}$ levels of the ${ }^{5} D_{4}$ multiplet. The two $\Gamma_{2}$ levels of ${ }^{5} D_{4}$ were found by examining the warm-up spectra. The experimental line positions of ${ }^{5} D_{4}$ are listed in Table IV.

\section{CALCULATIONS}

\section{A. Fitting procedure}

The energy levels of the ground LS term were calculated using the model described in Sec. IID. The six crystal-field parameters and the spinorbit parameter $\zeta$ were varied, until the best agreement with the 19 experimentally observed levels was obtained.

It is not relevant to let $F_{2}$ vary freely, since this parameter primarily must give the correct configuration splitting. However, the fitting was tried with several $F_{2}$ values between $434 \mathrm{~cm}^{-1}$ given by Wybourne ${ }^{18}$ and the "smoothed" value 417 $\mathrm{cm}^{-1}$ given by Karayianis. ${ }^{16}$ The result was not very sensitive to the $F_{2}$ value, but the best initial fit was obtained with $F_{2}=434 \mathrm{~cm}^{-1}$. This value was used in the remaining calculations.

In order to get an $L S$ term splitting in good agreement with the experiments, it was necessary to use an effective spin-orbit Hamiltonian including three terms $[p=3$ in Eq. (1)]. Note, however, that the number of fitting parameters is independent of the number of these terms. As experimental data for the ${ }^{7} F_{5}$ and ${ }^{7} F_{6}$ multiplets have not been 
obtained, it is especially important not to use more free parameters in the fitting procedure than dictated by the underlying physics.

Setting $\operatorname{Im} B_{64}$ equál to zero and fitting only five crystal-field parameters gave almost as good agreement as when all six crystal-field parameters were allowed to vary freely. This is what might be expected from the fact that the rare-earth ion is on a site of nearly $D_{2 d}$ symmetry, where all crystal-field parameters should be real. The final fittings, however, were done using all six crystal-field parameters.

\section{B. Results}

The fitting procedure was carried out at five temperatures between 10 and $100 \mathrm{~K}$. (For the lines only known experimentally at one temperature, a temperature dependence similar to the other lines was assumed.) As shown in Fig. 6 the crystalfield parameters were strongly temperature dependent. This was not the case for the $\zeta$ parameter. $\zeta$ was found to be $1698 \mathrm{~cm}^{-1}$ for all temperatures lower than $100 \mathrm{~K}$, where it was $1700 \mathrm{~cm}^{-1}$. The uncertainty of $\zeta$ was less than $2.5 \mathrm{~cm}^{-1}$. Wybourne ${ }^{18}$ gives a free-ion value of $1705 \mathrm{~cm}^{-1}$ for $\mathrm{Tb}^{3+}$. In crystals the spin-orbit coupling strength may differ from the free-ion value due to the coupling to the crystal field.

In the fittings a standard deviation of $12 \mathrm{~cm}^{-1}$ was obtained. The standard deviation is given by

$$
S=\left(\sum_{i=1}^{19} \frac{\left(\Delta E_{i}\right)^{2}}{19-6}\right)^{1 / 2} .
$$

The calculated energy levels at $10 \mathrm{~K}$ are given in Table III and the crystal-field parameters at $10 \mathrm{~K}$ in Table $\mathrm{V}$. The calculated position of the lowest $\Gamma_{3,4}$ level of the ground multiplet is 108 $\mathrm{cm}^{-1}$ at $10 \mathrm{~K}$ and $100 \mathrm{~cm}^{-1}$ at $100 \mathrm{~K}$. Holmes et al. ${ }^{2}$
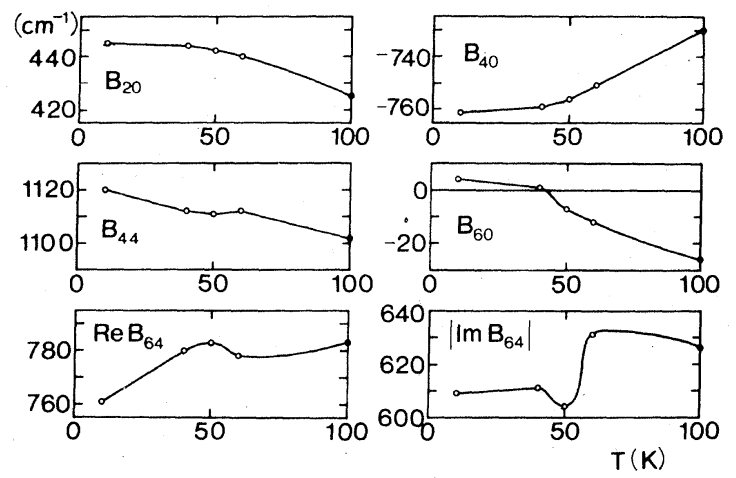

FIG. 6. Crystal-field parameters at the $T b^{3 *}$ site in $\mathrm{LiTbF}_{4}$ as a function of temperature. The sign of $\operatorname{Im} B_{64}$ is unknown. The lines through the points are only for visual aid.
TABLE V. Crystal-field parameters in $\mathrm{cm}^{-1}$. It is not possible to determine the sign of $\operatorname{Im} B_{64}$ with the methods referred to here. The first column gives the values obtained in this work, when the parameters are fitted inside the ${ }^{7} F$ term. The uncertainties correspond for the given model to an uncertainty on the experimental data of about $5 \mathrm{~cm}^{-1}$. The second column gives the values obtained by Holmes et al . (Ref. 2) by neutron-scattering and by susceptibility measurements. The third column gives the values obtained in this work, when the parameters are fitted inside the ${ }^{7} F_{6}$ multiplet.

\begin{tabular}{cccc}
\hline \hline & $\begin{array}{c}\text { Fitted to the } \\
{ }^{\eta_{F}} \text { term at } \\
10 \mathrm{~K}\end{array}$ & $\begin{array}{c}\text { Fitted to the } \\
\text { susceptibility }\end{array}$ & $\begin{array}{r}\text { Fitted to the }{ }^{7} F_{6} \\
\text { multiplet at } 10 \mathrm{~K}\end{array}$ \\
\hline$B_{20}$ & $445 \pm 10$ & $316 \pm 30$ & 474 \\
$B_{40}$ & $-761 \pm 30$ & $-673 \pm 130$ & -433 \\
$B_{44}$ & $1120 \pm 40$ & $628 \pm 130$ & 1080 \\
$B_{60}$ & $4 \pm 200$ & $-338 \pm 250$ & 64 \\
$\operatorname{Re} B_{64}$ & $761 \pm 130$ & $82 \pm 60$ & 744 \\
$\left|\operatorname{Im} B_{64}\right|$ & $609 \pm 130$ & $92 \pm 60$ & 286 \\
\hline \hline
\end{tabular}

find the same levels at 106 and $98 \mathrm{~cm}^{-1}$, respectively, by inelastic neutron scattering. Good agreement is also found for the next $\Gamma_{3,4}$ level, where the calculated values are 175 and $165 \mathrm{~cm}^{-1}$, and the experimental values from the inelastic neutron scattering are 169 and $161 \mathrm{~cm}^{-1}$.

\section{DISCUSSION}

\section{A. Crystal field}

As shown in Table $\mathrm{V}$ the spectroscopically determined $B$ parameters at $10 \mathrm{~K}$ differ rather much from those found by Holmes et al. ${ }^{2}$ by fitting to the susceptibility in the temperature range $5-300 \mathrm{~K}$ and to a few levels of the ground multiplet. The temperature dependence found (Fig. 6) indicates that a detailed comparison between these two sets of parameters cannot be undertaken.

The spectroscopically determined $B$ parameters give splittings of the ground multiplet-when diagonalizing within the whole $L S$ term-which is in good agreement with the seven lowest levels, which it has been possible to determine experimentally. As seen from Fig. 7 the calculated positions of both the remaining three high-lying levels as well as the seven lower-lying levels are in semiquantitative agreement with the experimental splittings for $\mathrm{Tb}^{3+}$ doped in $\mathrm{CaWO}_{4}$ given by Wortman. ${ }^{19}$

On the other hand, when diagonalizing only within the ground multiplet, but with $B$ parameters found by fitting to the whole $L S$ term, some of the levels come out wrong, differing as much as 50 $\mathrm{cm}^{-1}$ from the experimental values. This indicates 


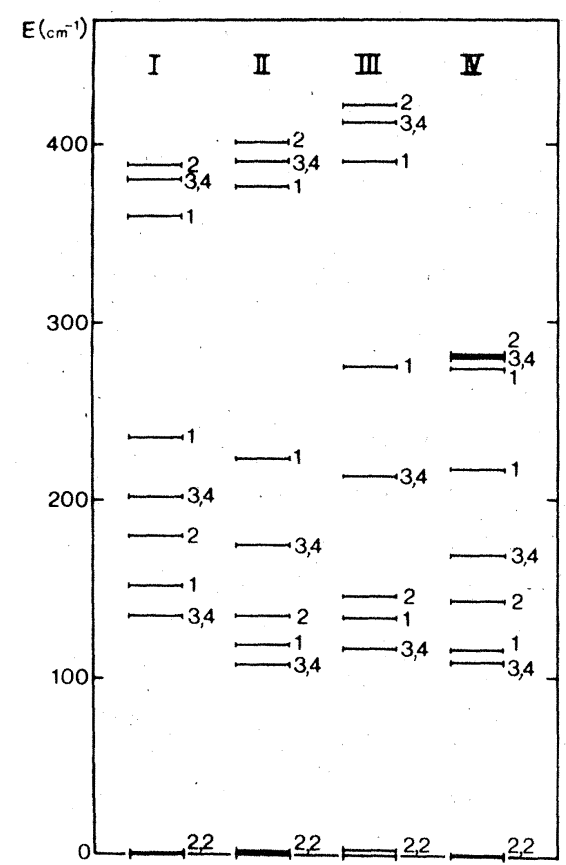

FIG. 7. Splittings of the ground multiplet of $\mathrm{Tb}^{3+}$. (I) Experimentally determined for $\mathrm{Tb}^{3+}$ doped in $\mathrm{CaWO}_{4}$ (Ref. 19). (II) Calculated for $\mathrm{Tb}^{3+}$ in $\mathrm{LiTbF}_{4}$ by diagonalizing the entire $L S$ matrix of the ground term. (III) Calculated for $\mathrm{Tb}^{3+}$ in $\mathrm{LiTbF}_{4}$ with the same parameters as in II, but diagonalizing only the $J$ matrix of the ground multiplet. (IV) Calculated for $\mathrm{Tb}^{3+}$ in $\mathrm{LiTbF}_{4}$ with the parameters of Holmes et al. (Ref. 2).

that even for the ground multiplet, $J$ mixing is significant. If the $B$ parameters are fitted inside the ground multiplet only, the $B$ values given in the third column of Table $\mathrm{V}$ are obtained. (Because of six parameters being available for fitting of 10 levels, the fit is perfect to within 1 $\mathrm{cm}^{-1}$.) It is clearly seen that the set of $B$ parameters are model dependent to a degree which is significant in relation to a quantitative discussion of experimentally determined properties. Still, it is remarkable that the order of all the levels is the same for all four cases depicted in Fig. 7 . This must be due to the circumstance that the different interactions are of such relative strengths that the $L S$ description as well as the $J$ description are both good enough for a quantitatively correct discussion of at least the lowest multiplet.

When the splitting of the ground multiplet is calculated using the parameters given by Holmes et al. ${ }^{2}$ it is clear from Fig. 7 that the three highest levels have too low energies, whereas the others fit very well.

The last fact is mainly due to the clamping of the lowest $\Gamma_{3,4}$ levels to the positions obtained by the neutron-scattering results, whereas the low value of the total splitting is connected to the low value of $B_{20} \cdot B_{20}$ is, however, almost tied to the difference between the reciprocal susceptibilities $\chi_{11}^{-1}$ and $\chi_{\perp}^{-1}$ at "high" temperatures. At $300 \mathrm{~K}$ this high-temperature limit is approached, and therefore the value of $B_{20}$, obtained by Homes et al., ${ }^{2}$ can be regarded as approximately correct for $T$ $\simeq 300 \mathrm{~K}$. In this connection it is noteworthy that if the temperature dependence of $B_{20}$ observed in the spectroscopic results persists up to room temperature, the value of $B_{20}$ will be lowered to about $360 \mathrm{~cm}^{-1}$ at this temperature. Yet, because of the significant but partly unknown temperature variation of all the $B$ 's, we shall defer at this stage to make a detailed comparison with the susceptibility data in the whole temperature range 4-300 K.

The calculated splitting $\Delta$ in the paramagnetic phase of the two $\Gamma_{2}$ levels spanning the ground quasidoublet is $1.9 \mathrm{~cm}^{-1}$, which is higher than the values given by Holmes et al. ${ }^{2}\left(1.3 \mathrm{~cm}^{-1}\right)$, by Magariño et al. ${ }^{11}\left(\simeq 1 \mathrm{~cm}^{-1}\right)$, and (for $\mathrm{Tb}^{3+}$ diluted in $\left.\mathrm{LiYF}_{4}\right)$ by Laursen and Holmes ${ }^{17}\left(0.93 \mathrm{~cm}^{-1}\right)$. Calculation with $B$ parameters fitted inside the ground multiplet gives a value of $1.5 \mathrm{~cm}^{-1}$ for $\Delta$.

The splitting $\Delta$ is, for $\mathrm{Tb}^{3+}$ in a Scheelite-crystal, strongly sensitive primarily to $\operatorname{Im} B_{64}$. If a value of $\Delta \simeq 1 \mathrm{~cm}^{-1}$ is considered the most reliable, this will therefore point to a somewhat lower value of $\operatorname{Im} B_{64}$ than that obtained from the term fitting.

\section{B. Temperature dependence of the spectrum}

Four features concerning the temperature dependence of the level positions have been found experimentally. (i) A small shift of the lines takes place in a narrow temperature region around $50 \mathrm{~K}$. (ii) The crystal-field splittings show a large continuous decrease in the temperature region $50-$ $100 \mathrm{~K}$. There is indication that this decrease continues to higher temperatures. (iii) The temperature effects are much less pronounced in a diluted system. (iv) At no temperature do the data indicate that additional crystal-field parameters are necessary in order to explain the level positions. This means that a transition to a lower rare-earth site symmetry is very unlikely.

Als-Nielsen et $a l .{ }^{7}$ have determined the structure of $\mathrm{LiTbF}_{4}$ at $100 \mathrm{~K}$ and at room temperature by neutron diffraction. They find that the crystal structure is very stable, although one coordinate of the fluorine ions is changed about $0.25 \%$ from $100 \mathrm{~K}$ to room temperature. The thermal expansion is small and anisotropic. The structure has not been determined below $100 \mathrm{~K}$, where the spectroscopic data indicate that something more inter- 
esting happens.

In the scheelite structure (space group $I 4_{1} / a$ ) there exist three degrees of freedom in the fluorine positions. The first fluorine can-apart from physical constraints - be placed arbitrarily in the unit cell, whereas the remaining fluorines are fixed by the symmetry requirements. Further the fluorines probably give the dominant contribution to the crystal-field energy of the $4 f$ electrons of the $R^{3+}$ ions. This coupling, together with the possibility of changing the fluorine positions without breaking the space-group symmetry, should in general (i.e., for all $\mathrm{Li} R \mathrm{~F}_{4}$ systems) lead to temperature-dependent properties. The magnitude of the effect is difficult to predict, but qualitatively a smooth temperature dependence might be expected.

The temperature dependence of the experimental data for $\mathrm{LiTbF}_{4}$ indicates, however, the existence of a characteristic transition temperature $T_{t}$ around $50 \mathrm{~K}$. The nature of the transition is not clear. Speculatively, the continuous character of the transition and the constancy of the $B$ 's below $T_{t}$ might be caused by the fluorine system being trapped in positions corresponding to local $D_{2 d}$ symmetry at the $R^{3+}$ sites. This is possible without breaking the global $S_{4}$ symmetry of the $R^{3+}$ site or the space-group symmetry. However, there would still exist two degrees of freedom for configurational changes in the fluorine system. Finally the cooperative nature of the phenomena-which is indicated by the weakening of the effect in a diluted system - is understandable, because each fluorine ion makes significant contributions to the crystal-field energy on at least two $R^{3+}$ sites.

In contrast to the temperature dependence observed for $\mathrm{LiTbF}_{4}$, Battison et al..$^{9}$ found no temperature dependence for $\mathrm{Ho}^{3+}$ in $\mathrm{LiHoF}_{4}$ for those transitions which they examined.

\section{ACKNOWLEDGMENTS}

The author is very grateful to I. Laursen, who has grown the dense $\mathrm{LiTbF}_{4}$ crystal and to Dr. R. Nevald and Professor V. Frank for their ideas and suggestions during the work and their constructive criticism of the manuscript.
${ }^{1}$ L. M. Holmes, T. Johansson, and H. J. Guggenheim, Solid State Commun. 12, 993 (1973).

${ }^{2}$ L. M. Holmes, H. J. Guggenheim, and J. Als-Nielsen, Proc. Int. Conf. Magn. 6, 256 (1973).

${ }^{3} \mathrm{~J}$. Als-Nielsen, L. M. Holmes, and H. J. Guggenheim, Phys. Rev. Lett. 32, 610 (1974).

${ }^{4}$ L. M. Holmes, J. Als-Nielsen, and H. J. Guggenheim, Phys. Rev. B 12, 180 (1975).

${ }^{5}$ P. E. Hansen, T. Johansson, and R. Nevald, Phys. Rev. B 12, 5315 (1975).

${ }^{6}$ R. Nevald and P. E. Hansen, Physica (Utr.) B 86-88, 1443 (1977).

${ }^{7} \mathrm{~J}$. Als-Nielsen, L. M. Holmes, F. K. Larsen, and H. J. Guggenheim, Phys. Rev. B 12, 191 (1975).

${ }^{8}$ A. H. Cooke, D. A. Jones, J. F. A. Silva, and M. R. Wells, J. Phys. C 8 4083 (1975).

${ }^{9}$ E. Battison, A. Kasten, M. J. M. Leask, J. B. Lowry, and B. M. Wanklyn, J. Phys. C 8, 4089 (1975).

${ }^{10}$ P. Beavillain, J. P. Renard, and P. E. Hansen, J. phys. C (to be published).

${ }^{11} \mathrm{~J}$. Magariño, J. Tuchendler, and I. Laursen, Proceedings of the Nineteenth Congress Ampere, 1976 p. 177 (unpublished); J. Magariño, J. Tuchendler, and P. E. Hansen, Physica (Utr.) B 86-88, 1233 (1977).

${ }^{12}$ H. P. Jenssen, D. Castleberry, D. Gabbe, and A. Linz, IEEE J. Quantum Electron. 9, 665 (1973).

${ }^{13} \mathrm{~T}$. Skettrup and H. P. Christensen (unpublished). The lifetime of the green fluorescence from $\mathrm{Tb}^{3+}$ was measured for $\mathrm{Tb}^{3+}$ in $\mathrm{LiTb}_{0.01} \mathrm{Y}_{0.99} \mathrm{~F}_{4}, \mathrm{LiTb}_{0.1} \mathrm{Y}_{0.9} \mathrm{~F}_{4}$, and $\mathrm{LiTbF}_{4}$. The lifetime in the diluted crystals was $5 \mathrm{msec}$ and in the dense crystal $1.4 \mathrm{msec}$. For excitation was used a $\mathrm{N}_{2}$ laser with $10-\mathrm{nsec}$ pulses.

${ }^{14} \mathrm{G}$. H. Dieke, Spectra and Energy Levels of Rare Farth Ions in Crystals (Wiley, New York, 1969).

${ }^{15}$ G. F. Koster, J. O. Dimmock, R. G. Wheeler, and H. Statz, Properties of the Thirty-two Point Groups (MIT, Cambridge, Mass., 1963).

${ }^{16}$ N. Karayianis, J. Chem. Phys. 53, 2460 (1970).

${ }^{17}$ I. Laursen and L. M. Holmes, J. Phys. C $\underline{7}, 3765$ (1974).

${ }^{18} \mathrm{~B}$. G. Wybourne, Spectroscopic Properties of the

Pare Earths (Interscience, New York, 1965).

${ }^{19}$ D. E. Wortman, Phys. Rev. 175, 488 (1968). 